\title{
Time Strengthening of Crystal Nanocontacts
}

\author{
Juan J. Mazo, ${ }^{1}$ Dirk Dietzel, ${ }^{2}$ Andre Schirmeisen, ${ }^{2}$ J. G. Vilhena, ${ }^{3}$ and Enrico Gnecco ${ }^{4}$ \\ ${ }^{1}$ Departamento de Física de la Materia Condensada and Instituto de Ciencia de Materiales de Aragón, \\ CSIC-Universidad de Zaragoza, 50009 Zaragoza, Spain \\ ${ }^{2}$ Institute of Applied Physics, Justus-Liebig University Giessen, 35392 Giessen, Germany \\ ${ }^{3}$ Departamento de Física Teórica de la Materia Condensada, Universidad Autónoma de Madrid, 28049 Madrid, Spain \\ ${ }^{4}$ Otto Schott Institute of Materials Research (OSIM), Friedrich Schiller University Jena, 07743 Jena, Germany
}

(Received 27 October 2016; published 16 June 2017)

\begin{abstract}
We demonstrate how an exponentially saturating increase of the contact area between a nanoasperity and a crystal surface, occurring on time scales governed by the Arrhenius equation, is consistent with measurements of the static friction and lateral contact stiffness on a model alkali-halide surface at different temperatures in ultrahigh vacuum. The "contact ageing" effect is attributed to atomic attrition and is eventually broken by thermally activated slip of the nanoasperity on the surface. The combination of the two effects also leads to regions of strengthening and weakening in the velocity dependence of the friction, which are well-reproduced by an extended version of the Prandtl-Tomlinson model.
\end{abstract}

DOI: 10.1103/PhysRevLett.118.246101

The increase of static friction with the time of contact between two solid surfaces was recognized as a key mechanism in earthquake dynamics already in the late 1970s by Diederich et al. [1]. In that context, the time dependence of the static friction force $F_{\text {stat }}$ is expected to be logarithmic, as suggested by experimental observations on granite. These results triggered the development of so-called "rate-and-state" theories [2,3], according to which, in addition, the kinetic friction $F_{\text {kin }}$ should decrease logarithmically with the sliding velocity, if so-called contact ageing is present. An investigation of these effects on the nanoscale has begun only recently using atomic force microscopy (AFM) [4-10]. The logarithmic velocity weakening of the friction has been clearly recognized when silicon tips slide on $\mathrm{SiO}_{2}$ and $\mathrm{NaCl}$ surfaces in the range of few nanometers per second to few microns per second driving speeds and at low temperatures of a few tens of degrees kelvin [11,12]. This effect is contrasted by a velocity strengthening, which also occurs on a logarithmic scale and is caused by thermal vibrations leading to premature slip [11-18]. When the temperature is increased, a transition from velocity weakening to strengthening was observed on those materials $[11,12]$. To close the circle, the logarithmic time dependence of the static friction initially proposed by Diederich et al. [1] has been also recognized on the nanoscale in recent measurements by Li et al. [5] on oxidized silicon tips pressed against a silicon wafer in ambient conditions.

In this Letter we combine the previous observations and propose a general model for the contact ageing of a sharp nanotip sliding on a crystal surface. This is done by increasing the amplitude of the surface potential with time at the location occupied by the tip, and using the wellknown Prandtl-Tomlinson (PT) model for atomic-scale friction. As a result, not only is the static friction force found to increase, but also the effective lateral stiffness $k$ of the sliding system. This is explained by a growth of the interface between the tip and surface, which is attributed to diffusion of surface atoms at the tip apex. The model results are supported by a newly applied analysis of the lateral stiffness of previously performed experimental stick-slip friction data on $\mathrm{NaCl}$ in ultrahigh vacuum (UHV)[12].

In contrast to the lateral force, the stiffness $k$ is a parameter that is often neglected in experimental work. It corresponds to a series of lateral springs modeling the deformation of the contact region formed by the tip apex and sample volume beneath, $k_{\mathrm{con}}$, and the deformation associated to the torsion of the cantilever support, $k_{\text {sensor }}[19,20]$. In Fig. 1(a) we show how its average value $\langle k\rangle$ varies with the scan velocity $v$ in a representative series of friction force microscopy measurements on the $\mathrm{NaCl}(001)$ cleavage surface at different temperatures. This value was estimated from the peak in the histogram of the local slope in the lateral force curves, as seen in Fig. 1(b) [21]. As a result, $\langle k\rangle$ is found to decrease logarithmically with the velocity $v$ or, equivalently, to increase logarithmically with the time of contact between the tip and surface, $t_{c} \approx a / v$, where $a=0.25 \mathrm{~nm}$ is the repetition distance of the stick slip.

In the elastic contact between a spherical tip and a flat surface, the quantity $k_{\mathrm{con}}$ is expected to vary as the square root of the contact area $A_{\text {con }}$ [20]. Note that this hypothesis is weakened if the atomic-scale surface roughness of the tip apex is considered [23], but, as it is not possible to determine the atomic structure of the tip while scanning, we retain it in the rest of the discussion. Since $k_{\text {sensor }}$ $(\sim 25 \mathrm{~N} / \mathrm{m}) \gg k$, we can also assume that $k \propto A_{\mathrm{con}}^{1 / 2}$, so that Fig. 1(a) suggests that $A_{\text {con }}$ should also increase logarithmically with the time of contact. However, an unlimited growth of $A_{\text {con }}$ is not realistic. Since the normal force $F_{N}$ is kept constant in the measurements, the "ageing" 

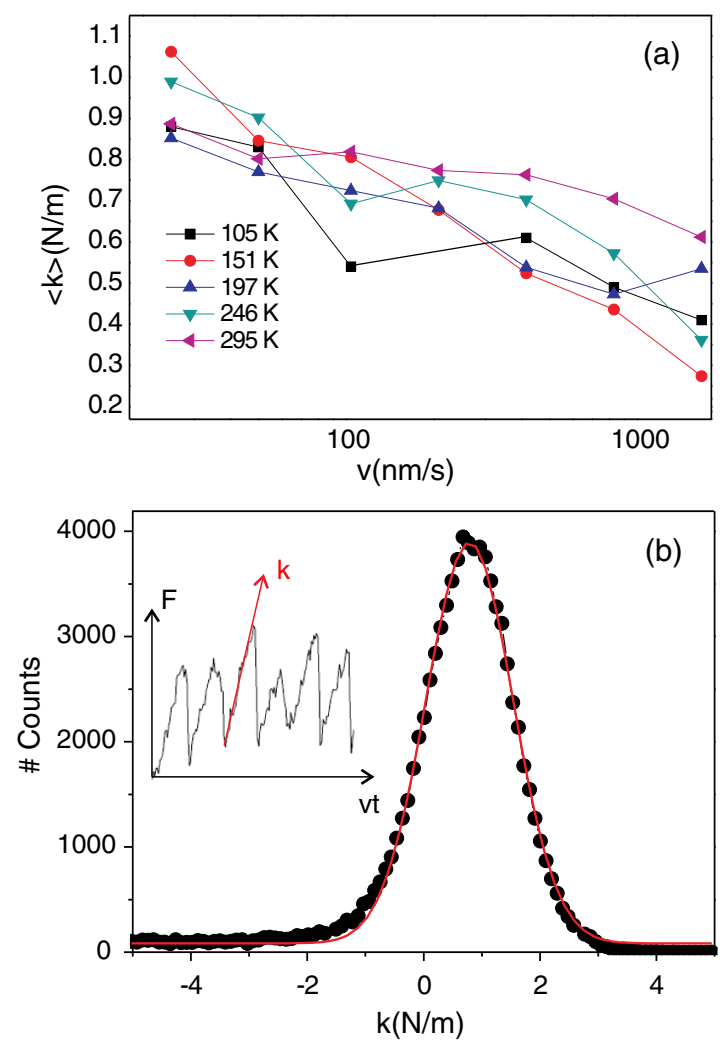

FIG. 1. (a) Average lateral stiffness $\langle k\rangle$ of the contact formed by an AFM tip sliding on a $\mathrm{NaCl}$ cleavage surface at different temperatures and scan velocities. The normal force $F_{N} \approx 5 \mathrm{nN}$. (b) Statistical distribution of the local slope of the lateral force (see inset) measured at $T=105 \mathrm{~K}$ and $v=50 \mathrm{~nm} / \mathrm{s}$. The derivative $d F / d x$ was estimated by three-point finite differences. The values corresponding to the peak of the Gaussian fit (red curve) are plotted as data points in (a).

process must end when the average pressure $p \sim F_{N} / A_{\text {con }}$ is on the order of the fracture strength of $\mathrm{NaCl}(\sim 3.5 \mathrm{MPa}$ [24]). For this reason, it is more reasonable to assume that the contact area saturates, e.g., exponentially with time, as

$$
A_{\text {con }}(t)=A_{0}[1+\alpha f(t)]
$$

where

$$
f(t)=1-\exp (-t / \tau)
$$

$A_{0}$ is the initial contact area, $\alpha$ is a constant that defines the maximum relative increase of $A_{\text {con }}$, and $\tau$ is a characteristic saturation time. In the following, Eq. (2) will be referred to as the ageing law of the contact. To support this assumption, we observe that a similar time dependence was observed in the lateral force variations recorded while repeatedly scraping a $\mathrm{KBr}$ surface in UHV with larger values of the normal force [25]. High resolution images of the damaged area, and specific features observed in atomicscale stick slip on $\mathrm{KBr}$ suggest that, in that case, the
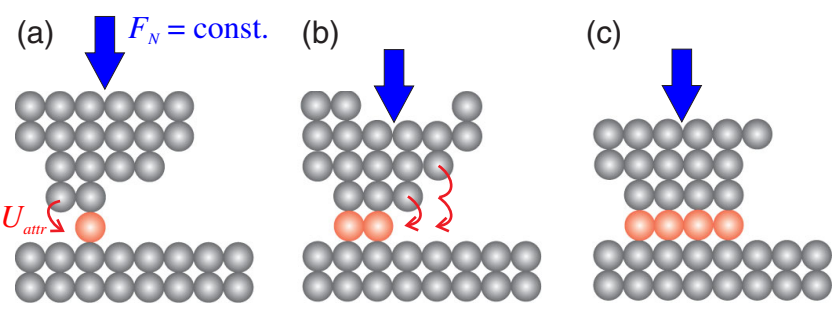

FIG. 2. Atomic attrition leading to contact ageing. (a) When a nanotip is pressed against a crystal surface, all pressure is initially carried by a contact that can be as small as a single atom. (b) To reach mechanical equilibrium atoms are flowing into the contact region, which increases the contact area and reduces the local pressure. (c) The process ends when the contact area is large enough to support the applied load without further atom movements.

pressure-induced surface diffusion of single atoms in the contact region and not plastic flow was responsible for the progressive disruption of the material. Because of the strong similarity between $\mathrm{KBr}$ and $\mathrm{NaCl}$, the same mechanism (which, following Bhaskaran et al. [26], may be called atomic attrition) should be also effective in the present case. Assuming that $k \propto A_{\text {con }}^{1 / 2}$, it is also expected that

$$
k(t)=k_{0} \sqrt{1+\alpha f(t)},
$$

where $k_{0}$ is the initial lateral stiffness.

As was first described by Prandtl [27], the stick slip of a nanotip on a crystal surface is caused by a periodic release of the elastic energy stored in the deformed contact into the bulk. The amount of this energy is determined by the amplitude of the tip-surface interaction potential $U_{\text {int }}$. Neglecting thermal vibrations, $U_{\text {int }}$ is proportional to the static friction $F_{s}$ [22]. Assuming the validity of Amonton's law, the latter will be also proportional to $A_{\text {con }}$, so that $U_{\text {int }}=U_{0}(1+\alpha f)$, with $U_{0}$ constant.

While the initial values and the maximum increments of $U_{\text {int }}$ and $k$ can be roughly inferred from the experiments, the characteristic time $\tau$ in Eq. (1) is more difficult to estimate. Assuming that atomic attrition is a thermally activated process, and following the extension of the Eyring model for polymer creep [28] proposed by Zhurkov [29] to describe general fracture processes, it makes sense to introduce the Arrhenius law

$$
\tau=\tau_{0} \exp \left(\frac{U_{\text {attr }}}{k_{B} T}\right)
$$

with a constant prefactor $\tau_{0}$, according to which $\tau$ decreases rapidly as the temperature increases [9]. In the schematic representation in Fig. 2 the energy $U_{\text {attr }}$ can be seen as the barrier preventing the slip of single atoms along the $\mathrm{NaCl}$ neck formed between the tip and surface. In a first approximation, $U_{\text {attr }}$ can be assumed on the same order 
of $U_{0}$ values close to contact breakup, when very few atoms form the interface between the tip and surface.

Here, the tip may be supposed to be coated by $\mathrm{NaCl}$, due to the prolonged scanning preceding the measurements and the considerable atom rearrangements frequently observed in this case [25,30]. Additional support for this hypothesis comes from MD simulations of AFM indentation showing that $\mathrm{MgO}$ tips are coated with $\mathrm{NaCl}$ when retracted from a $\mathrm{NaCl}$ slab [31]. Because of the electronegativity of the oxygen atoms, this is also expected with the $\mathrm{SiO}_{2}$ tips that we used. On the other side, this ageing mechanism should be absent on sample surfaces, where dynamic transfer and reformation of surface atoms at the tip apex is not possible. For this reason we also analyzed the lateral stiffness from previous atomic stick-slip measurements on HOPG [17]. In this case the layered nature of the sample surface does not allow atomic rearrangements and, indeed, we did not see any changes in a comparable experimental parameter space as on $\mathrm{NaCl}$ (see UHV Friction Measurements in Ref. [32], including Refs. [12,17,33,34]).

The value of $\tau_{0}$ can be estimated from the experimental variation of $\langle k\rangle$ with $v$ or, equivalently, with the time $t=a / v$, after fitting it with the relations (2)-(4). As a result, $\tau_{0} \sim 0.1 \mathrm{~ms}$, i.e., several orders of magnitude larger than the inverse of the Debye frequency, which is typically used as the prefactor for thermally activated atomic-scale processes. This can be attributed to the fact that atomic attrition involves the displacement of a multitude of atoms along the neck, and, since those atoms may jump not only in the preferential direction established by the tip pressure, the resulting creep is considerably slowed down.

In the rest of the Letter, the previous assumptions on the time variation of $U_{\text {int }}$ and $k$, and the temperature dependence of the ageing time $\tau$, are introduced in the PT model. The corresponding velocity dependence of the lateral force is evaluated numerically with the Langevin equation (as described in Numerical Simulation of Stick-Slip with Contact Ageing based on the Langevin Equation in Ref. [32], including Refs. [42,43]) and compared to the analytical expression given by Kramers's rate theory [35].

According to this theory, the probability that the tip remains pinned to the surface changes with time as [15]

$$
\frac{d p}{d t}=-f_{0}(t) \exp \left(-\frac{\Delta E(t)}{k_{B} T}\right) p(t)
$$

where $f_{0}$ and $\Delta E$ are a characteristic attempt frequency (see below) and, respectively, energy barrier. In our case, $\Delta E$ depends on the spring force $F \approx k v t$ as

$$
\Delta E=2 \sqrt{2} U_{\text {int }}\left(1-\frac{a F}{2 \pi U_{\text {int }}}\right)^{3 / 2}
$$

(assuming that $U_{\text {int }} \gg k a^{2}$ ) [36,37]. The most probable value of the static friction force at temperature $T$ and

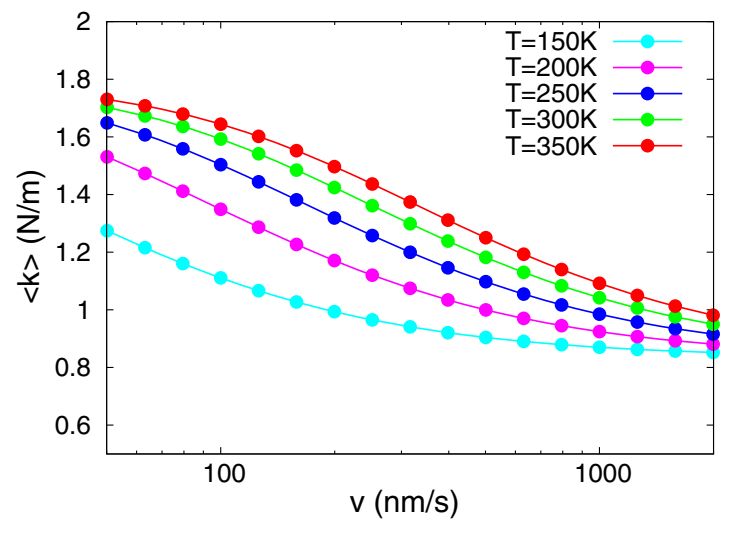

FIG. 3. Velocity weakening of the average value of the lateral contact stiffness $\langle k\rangle$, as estimated numerically from the PrandtlTomlinson model including contact ageing. Parameter values: $U_{0}=0.05 \mathrm{eV}, k_{0}=1 \mathrm{~N} / \mathrm{m}, a=0.25 \mathrm{~nm}, \alpha=3, \tau_{0}=0.1 \mathrm{~ms}$, $U_{\text {att }}=U_{0}, \gamma m=2 \times 10^{-6} \mathrm{~kg} / \mathrm{s}$, and $\sqrt{k_{0} / m}=2.5 \times 10^{6} \mathrm{~s}^{-1}$.

velocity $v, F_{S}^{*}(v, T)$, is obtained when the time variation of $p$ has a maximum. The frequency $f_{0}$ also changes with the force, and depends considerably on the effective damping of the system [38]. In our case $f_{0}$ can be approximated as

$$
f_{0} \approx \frac{\sqrt{2} \eta k}{2 \pi \gamma m} \sqrt{1-F / F_{s}^{0}},
$$

where $\eta=4 \pi^{2} U_{\text {int }} / k a^{2}, F_{s}^{0}=2 \pi U_{\text {int }} / a$, and the parameters $\gamma$ and $m$ are the effective damping and mass of the system, respectively. As a result, the following expression is expected [39]:

$$
F_{s}^{*}(v, T) \approx F_{s}^{0}-F_{s}^{0}\left(\frac{k_{B} T}{2 \sqrt{2} U_{\mathrm{int}}} \log \frac{v_{o}}{v}\right)^{2 / 3}
$$

with $v_{0} \approx \eta k_{B} T / 3 a \gamma m$. The same functional expression is found for the average static force $\left\langle F_{s}(v, T)\right\rangle$, where the $v_{0}$ term is now multiplied by a factor of $1.78[40,41]$. Note that Eq. (8) is obtained using the approximation $d F / d t=k(t) v$ in the time derivative of Eq. (6) and neglecting the slowly varying first time derivatives of $k(t), U_{\mathrm{int}}(t)$, and related quantities in Eqs. (5)-(7). These parameters are evaluated at the average time $\left\langle t_{c}\right\rangle$ when the first stick phase ends, which is estimated as described in Analytical Estimation of the First Slip Time at any Velocity and Temperature in Ref. [32] (including Refs. [42,43]) In all subsequent stick phases $\left\langle t_{c}\right\rangle$ is simply given by $a / v$ [44].

Figure 3 shows the velocity behavior of $\langle k\rangle$, as obtained by averaging consecutive values in Eq. (3) up to the slip time. $\langle k\rangle$ decreases logarithmically with the velocity, as observed in Fig. 1. It also increases slightly with $T$, although this effect is not discernible in the experiment. The velocity dependence of $\left\langle F_{s}\right\rangle$ at different temperatures is plotted in Fig. 4(a) for parameter values in the same orders of magnitude of the experiment (except for a broader 

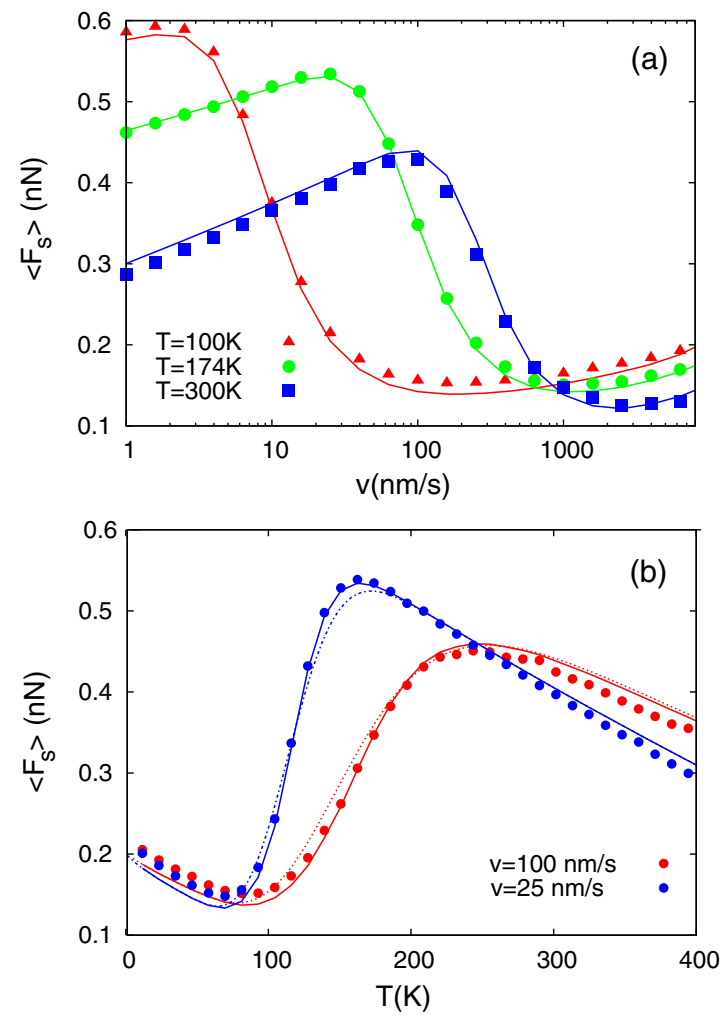

FIG. 4. (a) Velocity and (b) temperature dependence of $\left\langle F_{s}\right\rangle$ as estimated numerically by solving the Langevin equation (the points stand for the numerical result at first slip). The solid lines correspond to the theoretical prediction with $\left\langle t_{c}\right\rangle$ at the first slip. The dotted lines in (b) show the result for the contact time equal to $a / v$.

velocity range). The friction force increases logarithmically up to a certain value, where the effect of thermally activated slip is superseded by contact ageing and the trend is reversed (up to $v \approx a / \tau$ ). If the friction is recorded only in a limited "window" of velocities (e.g., around $v=50 \mathrm{~nm} / \mathrm{s}$ ) a slope reversal occurs with increasing $T$. This reversal was observed experimentally in Ref. [11] and attributed to thermally activated multibond dynamics at the interface. If, correspondingly, the $\left\langle F_{s}\right\rangle$ value at a given velocity $v$ in this window is plotted as a function of $T$, a friction peak is observed [Fig. 4(b)], which has been also reported experimentally [11]. Incidentally, a friction dip is also seen at low $T$, below the values accessible in the measurements. This is due to the fact that the ageing mechanism gets "frozen" at low $T$, as in this case the time $\tau$ becomes very large according to Eq. (4).

So far we have only limited the discussion to the static friction force. In the standard (thermally activated) PT model this is usually sufficient, since the kinetic friction force $F_{k}$ differs from $\left\langle F_{s}\right\rangle$ by a quantity close to $k a / 2$ [36]. However, the dynamics is more complex when contact ageing is taken into account. Here, we will simply assume that, when a contact is broken and the tip slips into the next pinning site, the new contact evolves again as shown in
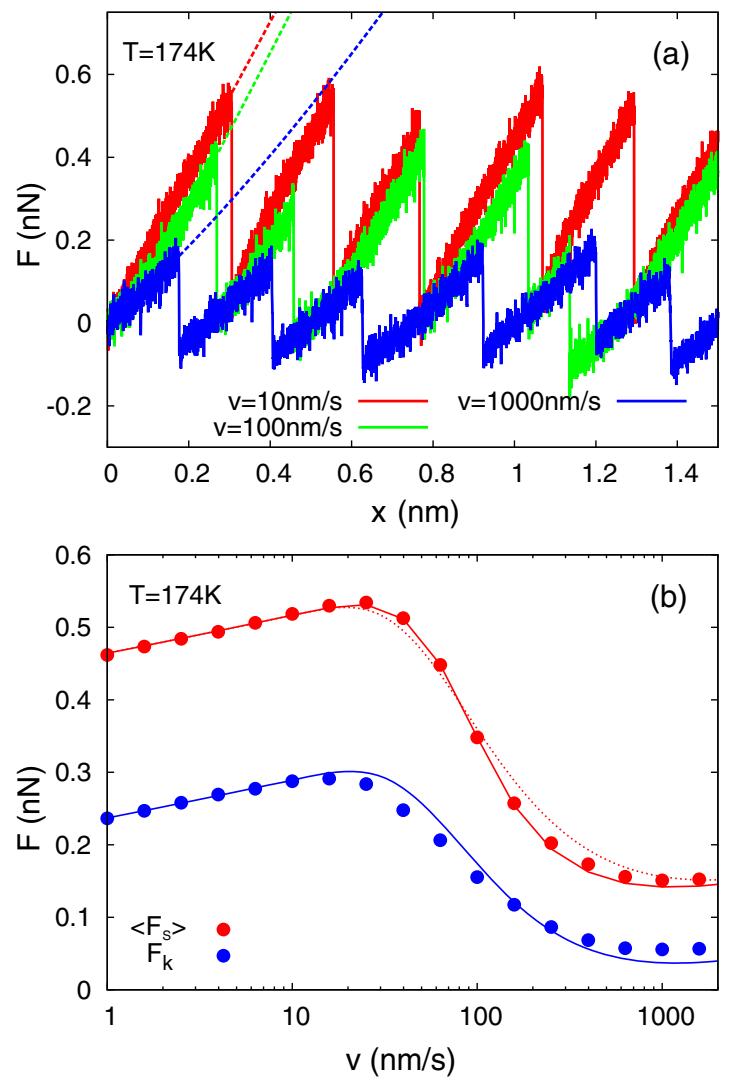

FIG. 5. (a) Lateral force versus support position for three different velocities. The dashed lines stand for the theoretical estimation $F(t) \simeq k(t) \eta(t) /[1+\eta(t)] v t$ [22]. (b) Static and kinetic friction as estimated using the Langevin equation (solid circles) and Kramers's theory (continuous lines). The solid and dotted lines refer to $\left\langle F_{s}\right\rangle$ values at first slip and subsequent ones.

Fig. 2(a). In other words, the parameters $U_{\text {int }}$ and $k$ are reset to the initial values $U_{0}$ and $k_{0}$, i.e., the contact is assumed to "rejuvenate," The resulting stick-slip profile is shown in Fig. 5(a). It is clear that the overall slope of the force curve in the stick phase increases at low velocity, as already shown in Fig. 3. The $F_{k}(v)$ and $\left\langle F_{s}(v)\right\rangle$ curves estimated in this way are compared in Fig. 5(b) to the expressions given by Kramers's rate theory, showing a good agreement with the last ones.

Summing up, the extended PT model that we propose is able to reproduce the three main effects observed in the experiments on a model $\mathrm{NaCl}(001)$ surface in UHV: (i) a slope reversal in the velocity dependence of the friction, (ii) a temperature peak of the friction, and (iii) a velocity weakening of the contact stiffness. A phenomenological ageing law has been introduced to describe the time variation of both the surface potential $U_{\text {int }}$ and the lateral contact stiffness $k$, which, under the simplest assumptions (Amonton's law, continuum contact mechanics), is proportional to $U_{\text {int }}^{1 / 2}$. As a reference system to test our approach we have studied a $\mathrm{NaCl}(001)$ crystal surface in UHV and have assumed that the $\mathrm{Na}$ and $\mathrm{Cl}$ ions diffuse in a barrier 
hopping motion along the neck formed between the tip and surface. This suggests an exponentially saturating ageing law, which is used in a series of numeric calculations. The main conclusion is that all experimental observations are reproduced by such a combination of the Prandtl model, with an elastically coupled lateral force and "degenerate final states, and of the Eyring model with a constant normal force and "nondegenerated" initial and final states [45] with a characteristic time defined by the ageing law. Extensions to different materials are possible by using different expressions for the ageing law $f(t)$ in Eqs. (1) and (3). Amorphous materials may be also included by introducing a proper distribution of values of the slip length $a$.

The authors acknowledge support from European Cooperation in Science and Technology (COST) Action MP1303. J. J. M. acknowledges Spanish Ministerio de Economía, Industria y Competitividad (MINECO) Project No. FIS2014-55867-P, cofinanced by Fondo Europeo de Desarrollo Regional (FEDER) funds, and Gobierno de Aragón Grant No. E19 to the FENOL group. D. D. and A. S. acknowledge German Research Foundation (DFG) Grants No. DI917/5-1 and No. SCHI619/10-1.

[1] J. Dieterich, J. Geophys. Res. 84, 2161 (1979).

[2] A. I. Ruina, J. Geophys. Res. 88, 10359 (1983).

[3] T. Baumberger, and C. Caroli, Adv. Phys. 55, 279 (2006).

[4] M. Evstigneev, A. Schirmeisen, L. Jansen, H. Fuchs, and P. Reimann, J. Phys. Condens. Matter 20, 354001 (2008).

[5] Q. Li, T. E. Tullis, D. Goldsby, and R. W. Carpick, Nature (London) 480, 233 (2011).

[6] N. N. Gosvami, M. Feldmann, J. Peguiron, M. Moseler, A. Schirmeisen, and R. Bennewitz, Phys. Rev. Lett. 107, 144303 (2011).

[7] Y. Liu and I. Szlufarska, Phys. Rev. Lett. 109, 186102 (2012).

[8] M. Evstigneev and P. Reimann, Phys. Rev. X 3, 041020 (2013).

[9] M. Feldmann, D. Dietzel, H. Fuchs, and A. Schirmeisen, Phys. Rev. Lett. 112, 155503 (2014).

[10] M. Feldmann, D. Dietzel, A. Tekiel, J. Topple, P. Grutter, and A. Schirmeisen, Phys. Rev. Lett. 117, 025502 (2016).

[11] I. Barel, M. Urbakh, L. Jansen, and A. Schirmeisen, Phys. Rev. Lett. 104, 066104 (2010).

[12] I. Barel, M. Urbakh, L. Jansen, and A. Schirmeisen, Phys. Rev. B 84, 115417 (2011).

[13] T. Bouhacina, J. P. Aime, S. Gauthier, D. Michel, and V. Heroguez, Phys. Rev. B 56, 7694 (1997).

[14] E. Gnecco, R. Bennewitz, T. Gyalog, C. Loppacher, M. Bammerlin, E. Meyer, and H. J. Guntherodt, Phys. Rev. Lett. 84, 1172 (2000).

[15] E. Riedo, E. Gnecco, R. Bennewitz, E. Meyer, and H. Brune, Phys. Rev. Lett. 91, 084502 (2003).
[16] S. Sills and R. M. Overney, Phys. Rev. Lett. 91, 095501 (2003).

[17] L. Jansen, H. Holscher, H. Fuchs, and A. Schirmeisen, Phys. Rev. Lett. 104, 256101 (2010).

[18] Q. Li, Y. Dong, D. Perez, A. Martini, and R. W. Carpick, Phys. Rev. Lett. 106, 126101 (2011).

[19] R. W. Carpick, D. F. Ogletree, and M. Salmeron, Appl. Phys. Lett. 70, 1548 (1997).

[20] M. A. Lantz, S. J. OShea, M. E. Welland, and K. L. Johnson, Phys. Rev. B 55, 10776 (1997).

[21] Note that in this approach $k$ differs by a small factor from the effective stiffness previously defined [22], which has almost no influence on the logarithmic trend that we observe.

[22] A. Socoliuc, R. Bennewitz, E. Gnecco, and E. Meyer, Phys. Rev. Lett. 92, 134301 (2004).

[23] B. Luan, and M. O. Robbins, Nature (London) 435, 929 (2005).

[24] G. Y. Akimov, and I. Y. Prokhorov, Phys. Status Solidi A 79, 423 (1983).

[25] E. Gnecco, R. Bennewitz, and E. Meyer, Phys. Rev. Lett. 88, 215501 (2002).

[26] H. Bhaskaran, B. Gotsmann, A. Sebastian, U. Drechsler, M. A. Lantz, M. Despont, P. Jaroenapibal, R. W. Carpick, Y. Chen, and K. Sridharan, Nat. Nanotechnol. 5, 181 (2010).

[27] L. Prandtl, ZAMM 8, 85 (1928).

[28] A. Tobolsky, and H. Eyring, J. Chem. Phys. 11, 125 (1943).

[29] S. N. Zhurkov, Int. J. Fract. 26, 295 (1984).

[30] P. E. Sheehan, Chem. Phys. Lett. 410, 151 (2005).

[31] S. Kawai, F. F. Canova, T. Glatzel, A. S. Foster, and F. Meyer, Phys. Rev. B 84, 115415 (2011).

[32] See Supplemental Material at http://link.aps.org/ supplemental/10.1103/PhysRevLett.118.246101.

[33] J. E. Sader, I. Larson, P. Mulvaney, and L. R. White, Rev. Sci. Instrum. 66, 3789 (1995).

[34] P. Bilas, L. Romana, B. Kraus, Y. Bercion, and J. L. Mansot, Rev. Sci. Instrum. 75, 415 (2004).

[35] H. Kramers, Physica (Utrecht) 7, 284 (1940).

[36] E. Gnecco, R. Roth, and A. Baratoff, Phys. Rev. B 86, 035443 (2012).

[37] O. K. Dudko, A. E. Filippov, J. Klafter, and M. Urbakh, Chem. Phys. Lett. 352, 499 (2002).

[38] J. J. Mazo, O. Y. Fajardo, and D. Zueco, J. Chem. Phys. 138, 104105 (2013).

[39] Y. Sang, M. Dube, and M. Grant, Phys. Rev. Lett. 87, 174301 (2001).

[40] O. K. Dudko, G. Hummer, and A. Szabo, Phys. Rev. Lett. 96, 108101 (2006).

[41] R. W. Friddle, Phys. Rev. Lett. 100, 138302 (2008).

[42] E. Helfand, Bell Syst. Tech. J. 58, 2289 (1979).

[43] H. S. Greensidea, and E. Helfand, Bell Syst. Tech. J. 60, 1927 (1981).

[44] For the parameter values used in the Letter $\left\langle t_{c}\right\rangle$ is very close to $a / v$ and thus the maximum force at the first peak is similar to the maximum force at other peaks [see Figs. 4(a) and 4(b)]. This is not the case in general.

[45] H. Spikes, and W. Tysoe, Tribol. Lett. 59, 21 (2015). 